\title{
Regional distribution of Christensenellaceae and its associations with metabolic syndrome based on a population- level analysis
}

\author{
Xiang Li ${ }^{\text {Equal first author, } 1}$, Zewen Li ${ }^{\text {Equal first author, } 1}$, Yan He ${ }^{1}$, Pan Li $^{1}$, Hongwei Zhou ${ }^{\text {Corresp., } 1}$, Nianyi Zeng ${ }^{\text {Corresp. } 1}$ \\ ${ }^{1}$ Microbiome Medicine Center, Division of Laboratory Medicine, Zhujiang Hospital, Southern Medical University, Guangzhou, Guangdong province, China
} Corresponding Authors: Hongwei Zhou, Nianyi Zeng

Email address: hzhou@smu.edu.cn, zengny1@i.smu.edu.cn

The link between the gut microbiota and metabolic syndrome (MetS) has attracted widespread attention. Christensenellaceae was recently described as an important player in human health, while its distribution and relationship with MetS in Chinese population is still unknown. This study sought to observe the association between Christensenellaceae and metabolic indexes in a large sample of residents in South China. A total of 4781 people from the GGMP project were included, and the fecal microbiota composition of these individuals was characterized by 16S rRNA sequencing and analyzed the relation between Christensenellaceae and metabolism using QIIME (Quantitative Insight Into Microbial Ecology, Version 1.9.1). The results demonstrated that microbial richness and diversity were increased in the group with a high abundance of Christensenellaceae, who showed a greater complexity of the co-occurrence network with other bacteria than residents who lacked Christensenellaceae. The enriched bacterial taxa were predominantly represented by Oscillospira, Ruminococcaceae, RF39, Rikenellaceae and Akkermansia as the Christensenellaceae abundance increased, while the abundances of Veillonella, Fusobacterium and Klebsiella were significantly reduced. Furthermore, Christensenellaceae was negatively correlated with the pathological features of MetS, such as obesity, hypertriglyceridemia and body mass index (BMI). We found reduced levels of lipid biosynthesis and energy metabolism pathways in people with a high abundance of Christensenellaceae, which may explain the negative relationship between body weight and Christensenellaceae. In conclusion, we found a negative correlation between Christensenellaceae and MetS in a large Chinese population and reported the geographical distribution of Christensenellaceae in the GGMP study. The association data from this population-level research support the investigation of strains within Christensenellaceae as potentially beneficial gut microbes. 
1 Regional distribution of Christensenellaceae and its

2 associations with metabolic syndrome based on a

3 population-level analysis

4

5 Xiang $\mathrm{Li}^{1 *}$, Zewen $\mathrm{Li}^{1}{ }^{*}$, Yan $\mathrm{He}^{1}$, Pan $\mathrm{Li}^{1}$, Hongwei Zhou${ }^{1}$, Nianyi Zeng ${ }^{1}$

6

$7 \quad{ }^{1}$ Microbiome Medicine Center, Division of Laboratory Medicine, Zhujiang Hospital, Southern

8 Medical University, Guangzhou, China.

$9 \quad *$ These authors contributed equally to this work.

10

11 Corresponding Author:

12 Hongwei Zhou ${ }^{1}$, Nianyi Zeng ${ }^{1}$

13253 Industrial Avenue, Guangzhou, Guangdong Province, 510282, China

14 Email address: $\underline{\text { hzhou@smu.edu.cn; zengny1@i.smu.edu.cn }}$ 


\section{Abstract}

25 The link between the gut microbiota and metabolic syndrome (MetS) has attracted widespread 26 attention. Christensenellaceae was recently described as an important player in human health, 27 while its distribution and relationship with MetS in Chinese population is still unknown. This study sought to observe the association between Christensenellaceae and metabolic indexes in a large sample of residents in South China. A total of 4781 people from the GGMP project were included, and the fecal microbiota composition of these individuals was characterized by $16 \mathrm{~S}$ rRNA sequencing and analyzed the relation between Christensenellaceae and metabolism using QIIME (Quantitative Insight Into Microbial Ecology, Version 1.9.1). The results demonstrated that microbial richness and diversity were increased in the group with a high abundance of Christensenellaceae, who showed a greater complexity of the co-occurrence network with other bacteria than residents who lacked Christensenellaceae. The enriched bacterial taxa were predominantly represented by Oscillospira, Ruminococcaceae, RF39, Rikenellaceae and Akkermansia as the Christensenellaceae abundance increased, while the abundances of Veillonella, Fusobacterium and Klebsiella were significantly reduced. Furthermore, Christensenellaceae was negatively correlated with the pathological features of MetS, such as obesity, hypertriglyceridemia and body mass index (BMI). We found reduced levels of lipid

41 biosynthesis and energy metabolism pathways in people with a high abundance of

42 Christensenellaceae, which may explain the negative relationship between body weight and

43 Christensenellaceae. In conclusion, we found a negative correlation between

44 Christensenellaceae and MetS in a large Chinese population and reported the geographical 45 distribution of Christensenellaceae in the GGMP study. The association data from this 
46 population-level research support the investigation of strains within Christensenellaceae as

47 potentially beneficial gut microbes.

48

49 Introduction

50 Metabolic syndrome (MetS) is a disease that brings together a variety of metabolic disorders.

51 With economic development and the improvement of living standards, the incidence of MetS has

52 also gradually increased, making this disease a global public health problem. MetS is

53 characterized by abdominal obesity, dyslipidemia, hypertension, and elevated blood sugar, and it

54 currently affects approximately 20 to $30 \%$ of adults worldwide (Grundy 2008). There are several

55 factors involved in the development of the disease, including host genetic factors (Kraja et al.

56 2011), eating habits and sedentary lifestyle (He et al. 2018b), but the pathogenesis of MetS has

57 not been fully elucidated.

58 With technological development, the role of the microbiome in human health has been

59 increasingly emphasized. The gut microbiota impacts a range of human health conditions,

60 including metabolic processes, immune-related diseases and neurological disorders (2019; Festi

61 et al. 2014; Fung et al. 2017). Studies on MetS and the gut microbiota in mouse models have

62 shown that the development of MetS involves a combination of the gut microbiota, host genes

63 and diet (Ussar et al. 2015; Zmora et al. 2019). Specific gut microbiota, bacterial metabolic

64 pathways and their interactions with human health are new focuses of microbiome research

65 (Proctor 2019), and understanding of the microbiome is intended to pave the way for future

66 microbiological therapies (Douillard \& de Vos 2019). An increasing number of studies have

67 reported that probiotics in the intestinal tract can maintain intestinal homeostasis by regulating

68 glucose and lipid metabolism, inhibiting the inflammatory response, and improving metabolic 
69 disorders (Lau et al. 2019; Plaza-Díaz et al. 2017; Sanders et al. 2019), thus preventing MetS and 70 related complications.

71 In this study, we focused on a family of Firmicutes named Christensenellaceae, where the 72 type strain Christensenellaceae minuta was first isolated from the feces of a healthy Japanese 73 man in 2012 and named after the Danish microbiologist Henrik Christensen (Morotomi et al. 74 2012; Waters \& Ley 2019). Since this family of bacteria was recently isolated, little is known 75 about its biological function other than its association with the host and with other 76 microorganisms. Goodrich et al. found that Christensenellaceae accounted for $0.01 \%$ of human 77 feces from the UK Twins population (Goodrich et al. 2014). In data from China (Kong et al. 78 2016; Wang et al. 2015), South Korea (Kim et al. 2019) and Italy (Biagi et al. 2016), the bacteria 79 were found to be highly abundant in centenarians. Brooks et al. reported that American females 80 have a higher abundance of Christensenellaceae than American males (Brooks et al. 2018). In 81 addition, a study conducted in Amsterdam showed that the relative abundance of

82 Christensenellaceae is ethnicity specific (Brooks et al. 2018; Deschasaux et al. 2018). These 83 studies demonstrated that age, gender, and ethnicity are associated with the abundance of 84 Christensenellaceae. The relative abundance of Christensenellaceae in the intestine was found to 85 be inversely proportional to body mass index (BMI) (Fu et al. 2015; Goodrich et al. 2014; Oki et 86 al. 2016; Peters et al. 2018b) and exhibited a negative correlation with obesity and inflammatory 87 bowel disease (Braun et al. 2019; Gevers et al. 2014; Imhann et al. 2018).

88 We previously performed the Guangdong Gut Microbiome Project (GGMP) study, constructing 89 the largest intestinal microbiota database for Eastern countries to date. In the present study, we 90 selected the Christensenellaceae-related population from GGMP to find out the relationship 91 between Christensenellaceae and regional distribution, metabolic index and metabolic diseases. 
92 We also focused on the connection between sequential operational taxonomic unit (sub-OTU)

93 (Amir et al. 2017) members of Christensenellaceae and the metabolic index. We also utilized the

94 GGMP dataset to reveal the metabolic pathway signatures of Christensenellaceae. A better

95 understanding of the relationship between MetS and Christensenellaceae may lead to new

96 therapeutic approaches for MetS.

97

98 Materials \& Methods

99

100

101

102

103

104

\section{Data collection and criteria for MetS}

The data used in this analysis from the GGMP were described previously (He et al. 2018c). In brief, 9172 individuals were investigated, and 6896 people finished the survey and household sampling (2276 missing metadata). By further filtering the metadata, some individuals with sequence number less than 10000 were excluded from this analysis, so a total of 6879 individuals were retained. The GGMP employs an in-person questionnaire to collect individual metadata. To evaluate how Christensenellaceae members affect the metabolism and gut microbes, we also compiled information on whether the individuals had diabetes, dyslipidemia, and pre-diabetic symptoms.

MetS was diagnosed as described by the Joint Committee for Developing Chinese Guidelines on Prevention and Treatment of Dyslipidemia in adults, based on three of the following five criteria for participants: (1) waist circumference $>90 \mathrm{~cm}$ (male) or $>85 \mathrm{~cm}$ (female), (2) fasting blood glucose $(\mathrm{FBG}) \geq 6.1 \mathrm{mmol} / \mathrm{L}(110 \mathrm{mg} / \mathrm{dl})$ or previously diagnosed with diabetes, (3) triglyceride $(\mathrm{TG}) \geq 1.7 \mathrm{mmol} / \mathrm{L}(150 \mathrm{mg} / \mathrm{dl})$, (4) high-density lipoprotein (HDL) $<1.04 \mathrm{mmol} / \mathrm{L}$ (40 mg/dl), and (5) systolic/diastolic blood pressure (SBP/DBP) $\geq 130 / 85 \mathrm{mmHg}$ or previously diagnosed with high blood pressure. 


\section{Sample collection and DNA extraction}

117 Stool samplers, ice bags and ice boxes were provided to collect and store samples after the

118 questionnaire survey. After defecation, each participant recorded their Bristol stool score and

119 stored the sample in an ice bag. All the samples were stored in a freezer $\left(-18^{\circ} \mathrm{C}\right.$ to $\left.-20^{\circ} \mathrm{C}\right)$ for

120 less than 3 days and then transported to the research laboratory (Guangdong CDC) in a cold-

121 chain vehicle to maintain a low-temperature environment. Samples were transported and stored

122 at the research laboratory in $-80^{\circ} \mathrm{C}$ freezers until further processing.

123 A total of $200 \mathrm{mg}$ of each fecal sample was used for DNA extraction using the Fecal DNA

124 Bead Isolation Kit (Bioeasy, Shenzhen) according to the manufacturer's instructions. Before the

125 specimens were submitted to laboratory analysis, we prepared external standards to control for

126 potential batch effects because multiple technicians and machines were involved in sample

127 processing. Briefly, fecal samples were collected from three donors. For each donor, the samples

128 were manually homogenized to obtain an even mixture, divided into 200 tubes and stored at -80

$129{ }^{\circ} \mathrm{C}$. All stool samples were processed with identical protocols, including three external standards

130 for each batch. For each DNA sample, the bacterial 16S rRNA gene was amplified with the

131 following barcoded primers ( shown from 5' to 3'): V4F (GTGYCAGCMGCCGCGGTAA) and

132 V4R (GGACTACNVGGGTWTCTAAT) (Walters et al. 2016). The primers contained Illumina

133 adapters and a unique 8-nucleotide barcode. The PCR conditions included an initial denaturation

134 at $94{ }^{\circ} \mathrm{C}$ for $5 \mathrm{~min} ; 30$ cycles of denaturation at $94{ }^{\circ} \mathrm{C}$ for $30 \mathrm{~s}$, annealing at $52{ }^{\circ} \mathrm{C}$ for $30 \mathrm{~s}$, and

135 elongation at $72{ }^{\circ} \mathrm{C}$ for $45 \mathrm{~s}$; and a final extension at $72{ }^{\circ} \mathrm{C}$ for $5 \mathrm{~min}$. The products were

136 submitted for next-generation sequencing on an Illumina HiSeq 2500 platform using 500-cycle

137 version 2 reagent kits (Beijing Genome Institute, BGI, Beijing). 
139 Microbiome bioinformatic analysis

140 Raw sequence data were managed and analyzed using Quantitative Insights Into Microbial

141 Ecology software (QIIME, version 1.9.1)(Caporaso et al. 2010), and the sequences with Phred

142 quality scores below 20 were then discarded. The method for processing of sequences was

143 identical to that described in our previous reports (He et al. 2018c). PCoA based on unweighted

144 UniFrac distances comparing bacterial community structure of samples between G1 and G2.

145 Permutational multivariate analysis of variance (PERMANOVA) was carried out to measure

146 effect sizes and significance differences in beta diversity. The threshold of statistical significance

147 was set at $\mathrm{P}<0.05$. We carried out multivariate association analyses with linear modeling

148 (MaAsLin) as described by Morgan et al. (Morgan et al. 2012) to examine the relationship

149 between sequential taxonomic units and each of the MetS diagnostic factors and several related

150 diseases. Age was used as a confounder, and the false discovery rate was limited to 0.05 . The $\mathrm{R}$

151 package (ggtree)(Yu et al. 2018) was used to visualize the evolution tree data after multi-

152 sequence comparison with QIIME. Data plotting and statistical analyses were performed by R

153 (3.2.2) statistical software.

154

155 Ethics approval and consent to participate

156 The present study was approved by the Ethical Review Committee of the Chinese Center for

157 Disease Control and Prevention under approval notice no. 201519-A. Written consent was

158 obtained from all participants.

159

160 Statistical analysis

Peer] reviewing PDF | (2020:03:46878:3:1:NEW 22 Jun 2020) 
161 The significance of differences between two groups was resolved by the Wilcoxon rank-sum test.

162 Spearman's rank correlation test was applied to analyze the correlation between two variables.

163 The chi-square test was utilized to compare the ratios of two groups. P values less than or equal

164 to 0.05 were considered significant. The Benjamini and Hochberg method was used to modulate

165 the P value for multiple hypotheses.

166

167 Results

168

\section{The overall gut Christensenellaceae configuration of people in the GGMP}

169

Exploration of the effects of the gut microbiota requires data from studies performed with a

170

regionalized study design, comprehensive sampling and standardized experimental protocols.

171 The population included in the GGMP has been previously described (He et al. 2018c). In the

172 GGMP project, 6896 individuals were included according to our entry criteria, which followed

173 the guidelines of the Joint Committee for Developing Chinese Guidelines as mentioned in a

174 previous article (He et al. 2018b). In the GGMP study, totally 6896 samples were characterized

175 by $16 \mathrm{~S}$ rRNA gene sequencing, and more than 17083 quality-filtered sequences were obtained

176 through QIIME analyses.Based on our statistics, the family Christensenellaceae accounted for an

177 average of $0.11 \%$ of human fecal bacteria in the residents. Then, we grouped the individuals

178 based on the abundance of Christensenellaceae; in total, 3316 people had no

179 Christensenellaceae in their intestines (Group1, G1), and we selected the upper quartile

180 population as Group2 (G2, n=1465) according to Christensenellaceae abundance. The

181 abundance of Christensenellaceae in this study was bias distributed, the overall median value

182 was 0 , and the maximum value was $15.1 \%$. Statistics found that the average abundance of

183 Christensenellaceae in the G2 group was $0.49 \%$.To characterize the diversity and richness of the

Peer] reviewing PDF | (2020:03:46878:3:1:NEW 22 Jun 2020) 
184 bacterial community, the alpha indices, estimated by four different parameters, were analyzed for 185 each sample. As shown in Fig. S1, the diversity and richness estimators in the two cohorts were 186 significantly different. Compared with the G1 subjects, the G2 subjects had a significantly high 187 alpha diversity indexes, such as the Chao1, observed OTU, PD_whole tree and Shannon indexes $188(\mathrm{p}<0.001$ for each). To measure the degree of similarity of the fecal microbial communities, we 189 performed a principal coordinate analysis (Fig. 1A), and we found obvious differences between 190 the two groups ( $\mathrm{R} 2=0.07184, \mathrm{P}=0.001$ ). Additionally, we applied linear discriminant analysis 191 effect size (LEfSe) (Segata et al. 2011) for quantitative analysis of biomarkers within different 192 cohorts. A total of 20 features had significantly different abundances between G1 and 193 G2(LDA>3) (Fig. 1B). At the genus level, the fecal microbiota of people who lacked 194 Christensenellaceae was enriched with the taxa Proteobacteria, Enterobacteriales, 195 Bacteroidaceae, Lachnospiraceae and Klebsiella, whereas individuals with a high proportion of 196 Christensenellaceae exhibited enrichment of Ruminococcaceae, Mollicutes, RF39, Akkermansia 197 and Rikenellaceae.

198 To examine the Christensenellaceae components in detail, we analyzed the sub-OTUs at the 199 family level. After chimera removal and quality filtering, a total of 134 different sub-OTU of 200 Christensenellaceae were identified at the $100 \%$ sequence identity level, and eight of them had 201 read counts greater than 1000 (Fig. 1C). An evolutionary tree was constructed to further observe 202 the evolutionary distance of each sub-OTU and several reference genome (Fig. S1).

203

\section{The distribution of Christensenellaceae in GGMP}

205 In our survey for the GGMP study, the distribution of Christensenellaceae in Guangdong

206 Province varied based on geographic location. According to the average abundance data, 
207 Christensenellaceae is unevenly geographically distributed, with the abundance typically

208 reduced in or near large and economically thriving centers such as Guangzhou (0.08\%) and

209 Shenzhen $(0.09 \%)$. In contrast, Shanwei had the highest abundance $(0.22 \%)$, followed by

210 Huizhou (0.19\%) and Meizhou (0.18\%). The abundances in other cities is shown in Fig. 2A. In

211 addition, we collected data on per capita annual income and Christensnellaceae abundance in

212 each region (Fig. 2B). According to the map, the higher the level of urbanization, the lower the

213 abundance of Christensenellaceae in the intestinal tract of urban residents.

214 When the MetS prevalence in different cities was compared, there was a significantly negative 215 correlation between MetS and Christensenellaceae abundance (Fig. 2C). Residents in Zhanjiang, 216 Shanwei, Foshan and Maoming had Christensenellaceae abundances higher than $0.15 \%$ in their 217 gut and exhibited a low prevalence of MetS. Similarly, city dwellers in Guangzhou, Shenzhen 218 and Shaoguan had a low Christensenellaceae abundance, and a high proportion of the population 219 exhibited MetS. However, a negative correlation was not observed in residents in Jieyang and 220 Meizhou. Notably, the prevalence of MetS in G2 subjects was lower than that in G1subjects in 221 most regions (Fig. 2D). Moreover, there was a significant difference in the prevalence of MetS in 222 people with different abundances of Christensenellaceae in Qingyuan $(\mathrm{p}<0.01)$ and Shanwei $223(\mathrm{p}<0.05)$. Although Christensenellaceae abundance varies in different regions, the negative 224 correlation between MetS and Christensenellaceae is universal.

225

226

\section{Christensenellaceae is associated with host metabolic index and MetS status}

227 To explore the relationship between Christensenellaceae abundance and human health, we next 228 assessed how variable the body parameters were in terms of Christensenellaceae richness. We selected 4781 subjects associated with Christensenellaceae from the GGMP project, and subjects 
230 in this study cohort had a wide range of age, BMI and blood test indicators (Table 1). As

231 previously described, we classified the subjects into two categories (G1andG2) according to the

232 abundance of Christensenellaceae. The differences between the two groups were mainly

233 reflected in the following aspects: age, anthropometric parameters and biochemical criteria

234 (Table 1). Because variations in metabolites could be related to differences in age (Dunn et al.

235 2015), we reanalyzed the correlation between the indices after adjusting for age. Compared to the

236 G1 group, people rich in Christensenellaceae showed significantly low BMI, waist

237 circumference and waist-to-height ratio (WHtR). The high-value group also showed lower levels

238 of biochemical indices, such as TG, alanine transaminase (ALT) and uric acid (UA), than the

239 non-Christensenellaceae group (Fig. S2). The level of HDL was significantly higher in people

240 with Christensenellaceae, indicating the abundance of Christensenellaceae has a positive

241 correlation with HDL level.

242 In addition, we calculated the correlation coefficients between the waist circumference,

243 WHtR, BMI, TG level and other clinical parameters of all subjects to evaluate the connection

244 intensity between the abundance of Christensenellaceae and the host metabolic index (Fig. 3A).

245 We found that waist circumference and WHtR remained significantly decreased in people rich in

246 Christensenellaceae compared to the people who lacked Christensenellaceae. Recently, waist

247 circumference and WHtR were proposed as predictors of the incidence of MetS(Perona et al.

248 2019; Suliga et al. 2019). Therefore, we measured the prevalence of MetS in individuals with

249 GGMP. People rich in Christensenellaceae had low prevalence of metabolic diseases, including

250 overweight, obesity, fatty liver disease, and hypertriglyceridemia.

251 We also tested for connections between the sequential taxonomic units of Christensenellaceae

252 and the indicators to observe whether the changes in sub-OTUs at the family level of 
253 Christensenellaceae are consistent. These top eight sub-OTUs, with reads number greater than

254 1000, had the same correlation with each parameter (Fig. 3A). They all had negative correlations

255 with ALT, TG, UA, waist circumference and WHtR. Among the sub-OTUs, sub-OTU3228 had a 256 significantly negative correlation with TG, ALT, UA and BMI. Nevertheless, most of the 257 taxonomic units were positively related to HDL and blood urea nitrogen (BUN).

258 In addition to morphological indicators and circulating metabolites, we also identified an 259 association between the family Christensenellaceae and metabolic diseases such as fatty liver 260 disease, obesity, hypertriglyceridemia and digestive system disease (Fig. 3B). Moreover, we 261 found that sub-OTU3228, sub-OTU5291, sub-OTU12860, and sub-OTU14698 were negatively 262 correlated with overweight, obesity and hypertriglyceridemia. Although most of the associated 263 taxa were shared across obesity and lipid metabolites, several sequences were predominantly 264 linked to digestive diseases rather than metabolic disorders. Notably, the abundances of sub265 OTU3228 and sub-OTU14698 were negatively associated with digestive disorders, while sub266 OTU3228 showed a strong positive correlation with the occurrence of diabetes mellitus (T2DM). 267 Besides the above four members, the remaining taxonomic units with read counts greater than 2681000 were not significantly associated with these metabolism-related disorders.

\section{Specific microbial taxa associated with Christensenellaceae}

271 We used co-occurrence network analysis to investigate the interactions among the microbes in 272 the complex intestinal microbiota. After pairwise correlation analysis of the bacteria in all the 273 volunteers' stool samples, the major bacteria associated with Christensenellaceae in the 274 individuals enrolled in the study are shown in Fig. 4A. Of all the species, the abundances of 275 Veillonella, Ruminococcus, Fusobacterium, and Blautia were most highly negatively related to 
276 Christensenellaceae. Additionally, species such as Ralstonia and Klebsiella had a negative

277 correlation with the abundance of Christensenellaceae. In addition, we identified a significant

278 positive association of Christensenellaceae with Oscillospira, Ruminococcaceae, RF39,

279 Rikenellaceae and Akkermansia. Moreover, Lachnospiraceae, Roseburia and Sediminibacterium

280 also showed expected relationship with Christensenellaceae.

281 The focus was not only on the family level of the microbiota but also on the sub-OTUs of

282 Christensenellaceae. The correlation between sequential taxonomic units of Christensenellaceae

283 and other bacteria was universal. As shown in Fig. 4B, the eight sequences with read counts in

284 excess of a thousand were consistent with the relationships between other bacteria. The four

285 sequences sub-OTU14698, sub-OTU3228, sub-OTU12860, and sub-OTU5219 showed the

286 strongest correlation, and the predominant bacteria in the positive correlation included

287 Oscillospira, Clostridiales, RF39 and Rikenellaceae. Nevertheless, Veillonella, Fusobacterium,

288 Blautia, Megamonas and Streptococcus were negatively associated with these five sub-OTUs. In 289 general, the bacterial network relationship at the sequence level and the family level is basically 290 the same.

291

292 5. Functional properties predicted by PICRUSt

293 We performed PICRUSt analysis to predict the KEGG functional orthologs of the fecal

294 microbiota metagenomes based on 16S rRNA sequences. Principal component analysis (PCA)

295 showed that the KO profile of the gut microbiota in people with high abundance of

296 Christensenellaceae diverged from that of the gut microbiota of people lacking

297 Christensenellaceae (Fig. S3). $71.2 \%$ of the variation within these two groups was captured by

298 the first principal component (PC1). As shown in Fig. 5, broad potential communication 
299 pathways were identified between the individuals, including metabolism, cellular process,

300 environmental information process, genetic information process and human disease. The LEfSe

301 algorithm was applied to detect differences in the functional pathways of the microbiota between

302 the two groups. In total, 6 functional orthologs were significantly different in

303 Christensenellaceae-rich people (LDA score $>3$ ); the enriched orthologs were nucleotide

304 metabolism; cellular process; ribosome; translation; replication and repair; and genetic

305 information processing. In contrast, the prevalent markers among the controls included those

306 associated with transporters, membrane transport and environmental information processing

307 (Fig. 5). Moreover, several metabolic pathways were abundant in the non-Christensenellaceae

308 group, including those involved in energy metabolism (methane metabolism), lipid metabolism

309 (fatty acid biosynthesis) and carbohydrate metabolism (fructose and mannose metabolism)

310 (Table S1). In terms of metabolic disease, people with Christensenellaceae have a relatively low

311 risk of diabetes mellitus (Table S1). These findings suggest that Christensenellaceae may affect

312 the way in which we metabolize and regulate ponderal growth.

313

\section{Discussion}

315 This study was based on a rigorous experimental design and strict quality control, and fecal

316 samples and host information of 6896 volunteers were collected in Guangdong Province. The use

317 of 16S rRNA sequencing helped us clearly elucidate the complexity of the gut microbial

318 ecosystem. We could focus not only on the composition and distribution of intestinal bacteria but

319 also on the metabolic functions of these bacteria. Through the integration of these data, we could 320 focus on the associated OTUs classified within the Christensenellaceae family. And we can 
321 target these OTUs as a keystone and link the etiology and pathogenesis of MetS to intestinal

322 microorganisms to provide potential therapeutic targets.

323 First, the present study showed a variation in Christensenellaceae among residents in different

324 areas by comparing the average abundance for each region. Based on our previous research,

325 MetS prevalence was significantly higher in individuals with higher economic status than in

326 those of with lower economic status (He et al. 2018a). As we found that Christensenellaceae is

327 negatively correlated with MetS, it can be explained to some extent that people in economically

328 developed regions have lower intestinal abundance of Christensenellaceae. Moreover, the

329 individual differences in Christensenellaceae may simply be a result of environmental or genetic

330 factors (Waters \& Ley 2019). And our results for large study population showed that the negative

331 correlation between MetS and Christensenellaceae is consistent in most areas.

332 As previously reported, the abundance of Christensenella, Bifidobacterium and Akkermansia

333 has been recognized as a signature of the gut ecosystem for healthy aging and longevity (Biagi et

334 al. 2016; Derrien et al. 2017; Wang et al. 2015). We also found that people with a high

335 abundance of Christensenellaceae were older than those in the control group. Changing

336 functions of the intestine with age may affect the abundance of bacteria.

337 The microbiota inhabiting the intestinal cavity affects body health by altering the metabolome

338 and regulating the bacterial bioavailability of nutrients in the lumen. (Johnson et al. 2015; Liu et

339 al. 2018; Romano et al. 2015). In this study, we observed a significant association between the

340 gut microbiota and the variation in BMI and blood lipid levels, which is independent of age. We

341 observed that the abundance of Christensenellaceae was significantly related to the individual

342 variance in BMI and to the blood levels of TG and HDL but has not much correlation with low-

343 density lipoprotein (LDL) or total cholesterol (TC) levels. The analysis showed that people with 
344 higher Christensenellaceae abundance in their gut had lower BMI and lower TG levels. These

345 results are consistent with the previous report demonstrating that Christensenellaceae abundance

346 was negatively correlated with BMI and triglycerides and positively correlated with HDL levels

347 in the Dutch LifeLines DEEP cohort $(\mathrm{n}=893)$ and reports from other countries. (Fu et al. 2015;

348 Peters et al. 2018a; Waters \& Ley 2019). As low HDL levels are one of the criteria of metabolic

349 syndrome(Yang \& Wang 2019). The positive relationship between Christensenellaceae and

350 HDL suggests a potentially beneficial role in metabolism. Therefore, in the complex network of

351 indicators associated with Christensenellaceae, these findings show the negative correlation

352 between Christensenellaceae with the prevalence of MetS.

353 The microbial interaction network has been considered an important biological factor in the

354 occurrence and progression of metabolic system diseases. We observed significant microbial

355 community changes in people with high abundance of Christensenellaceae, in whom the

356 richness and diversity of the microbial community increased significantly. Our results highlight

357 the positive correlation between Christensenellaceae and Oscillospira, Ruminococcaceae, RF39,

358 Rikenellaceae and Akkermansia (Goodrich et al. 2014), while the bacteria were negatively

359 related to Veillonella, Fusobacterium and Klebsiella. Previous studies have shown that

360 Oscillospira and Ruminococcaceae are positively associated with health and leanness (Konikoff

361 \& Gophna 2016; Ziętak et al. 2016). Other noteworthy taxa include Akkermansia, RF39 and

362 Rikenellaceae, which have been experimentally confirmed to be probiotics and are considered

363 beneficial to limit high fat-induced body weight gain. (Alard et al. 2016; Wang et al. 2017).

364 Moreover, previous reports have shown that Fusobacterium and Klebsiella are positively

365 correlated with the levels of cholesterol and LDL and alter lipid metabolism (Fei et al. 2020;

366 Koren et al. 2011). How Christensenellaceae members impact the diversity and structure of the 
367 intestinal microbiota is still unclear. Nevertheless, it is plausible that changes in the intestinal

368 niche induced by Christensenellaceae can promote lipid metabolism and contribute to the

369 maintenance of normal body weight.

370 Our work showed differences in the predicted microbiota function in people with different

371 abundances of Christensenellaceae. Previous reports indicated that obesity markers were

372 typically positively associated with the KEGG categories of fructose metabolism (Hannou et al.

373 2018) and methane metabolism (Mathur et al. 2013), which are enriched in people who lack

374 Christensenellaceae. However, it's not a general consistency with the previous result that

375 Methanobacteriaceae increased in G2 group. PICRUSt represents the methane metabolism of the

376 whole gut flora, but the single increased abundance of Methanobacteriaceae does not indicate

377 the overall function. The high abundance of Christensenellaceae in individuals showed

378 decreased fatty acid biosynthesis. Increasing evidence has shown that fatty acid accumulation is

379 significantly associated with metabolic diseases and obesity(Sonnenburg \& Bäckhed 2016).. This

380 confirmed the previously observed negative correlation between Christensenellaceae and

381 metabolic indicators. To develop a deeper understanding of MetS progression, further follow-up

382 studies are required to examine the significance of microbial functional variations in body

383 metabolism. Furthermore, metatranscriptomic and metabolomic analyses could be used to

384 elucidate the detailed pathways of gene and metabolite interactions, enhancing the understanding 385 of the effects of intestinal bacteria.

386

387 Conclusions

388 In the present study, we used multivariate association analyses to explore the association

389 between Christensenellaceae and metabolic indexes in nearly 5000 participants in South China. 
390 Based on the results, Christensenellaceae is related to a low risk of MetS and obesity, showing 391 similarities to results obtained in Western populations. In addition, significant correlations were 392 found between Christensenellaceae and other intestinal bacteria through analysis of the bacterial 393 network. The bacteria that were positive correlated with Christensenellaceae were mainly 394 beneficial bacteria that had been identified previously, while some pathogenic bacteria were 395 negatively correlated with Christensenellaceae. Finally, PICRUSt was applied to analyze the 396 pathway differences caused by Christensenellaceae, which provided bioinformatics evidence for 397 predicting the correlation between Christensenellaceae and metabolic alterations in the gut 398 microbiota community. However, the underlying mechanisms through which 399 Christensenellaceae members regulate host metabolism need to be explored.

400

\section{Additional Information and Declarations}

402 Availability of data and materials

403 The raw data for 16S rRNA gene sequence is available from the European Nucleotide Archive 404 (https://www.ebi.ac.uk/ena/) at accession number PRJEB18535. The bioinformatics code used 405 for raw data processing is available form https://github.com/SMUJYYXB/GGMP.

408 Acknowledgments

409 We acknowledge the contributions of the 308 local CDC investigators and registered nurses, who 410 helped with collection point maintenance and metadata and stool sample collection. We thank all 411 the volunteers who participated in this project. 


\section{References}

414 Alard J, Lehrter V, Rhimi M, Mangin I, Peucelle V, Abraham A-L, Mariadassou M, Maguin E, 415 Waligora-Dupriet A-J, Pot B, Wolowczuk I, and Grangette C. 2016. Beneficial metabolic effects of selected probiotics on diet-induced obesity and insulin resistance in mice are

Amir A, McDonald D, Navas-Molina JA, Kopylova E, Morton JT, Zech Xu Z, Kightley EP, Thompson LR, Hyde ER, Gonzalez A, and Knight R. 2017. Deblur Rapidly Resolves Single-Nucleotide Community Sequence Patterns. mSystems 2. 10.1128/mSystems.00191-16

Biagi E, Franceschi C, Rampelli S, Severgnini M, Ostan R, Turroni S, Consolandi C, Quercia S, Scurti M, Monti D, Capri M, Brigidi P, and Candela M. 2016. Gut Microbiota and Extreme Longevity. Current biology : CB 26:1480-1485. 10.1016/j.cub.2016.04.016

Braun T, Di Segni A, BenShoshan M, Neuman S, Levhar N, Bubis M, Picard O, Sosnovski K, Efroni G, Farage Barhom S, Glick Saar E, Lahad A, Weiss B, Yablecovitch D, Lahat A, Eliakim R, Kopylov U, Ben-Horin S, and Haberman Y. 2019. Individualized Dynamics in the Gut Microbiota Precede Crohn's Disease Flares. The American journal of gastroenterology 114:1142-1151. 10.14309/ajg.0000000000000136

Brooks AW, Priya S, Blekhman R, and Bordenstein SR. 2018. Gut microbiota diversity across ethnicities in the United States. PLoS biology 16:e2006842.

Caporaso JG, Kuczynski J, Stombaugh J, Bittinger K, Bushman FD, Costello EK, Fierer N, Peña AG, Goodrich JK, Gordon JI, Huttley GA, Kelley ST, Knights D, Koenig JE, Ley RE, Lozupone CA, McDonald D, Muegge BD, Pirrung M, Reeder J, Sevinsky JR, Turnbaugh PJ, Walters WA, Widmann J, Yatsunenko T, Zaneveld J, and Knight R. 2010. 

7:335-336. 10.1038/nmeth.f.303

440 Derrien M, Belzer C, and de Vos WM. 2017. Akkermansia muciniphila and its role in regulating host functions. Microbial pathogenesis 106:171-181. 10.1016/j.micpath.2016.02.005

442 Deschasaux M, Bouter KE, Prodan A, Levin E, Groen AK, Herrema H, Tremaroli V, Bakker GJ, 443 Attaye I, Pinto-Sietsma S-J, van Raalte DH, Snijder MB, Nicolaou M, Peters R, Zwinderman AH, Bäckhed F, and Nieuwdorp M. 2018. Depicting the composition of gut

Douillard FP, and de Vos WM. 2019. Biotechnology of health-promoting bacteria. Biotechnology advances 37:107369.

Dunn WB, Lin W, Broadhurst D, Begley P, Brown M, Zelena E, Vaughan AA, Halsall A, Harding N, Knowles JD, Francis-Mclntyre S, Tseng A, Ellis DI, O'Hagan S, Aarons G, Benjamin B, Chew-Graham S, Moseley C, Potter P, Winder CL, Potts C, Thornton P, McWhirter C, Zubair M, Pan M, Burns A, Cruickshank JK, Jayson GC, Purandare N, Wu FCW, Finn JD, Haselden JN, Nicholls AW, Wilson ID, Goodacre R, and Kell DB. 2015. Molecular phenotyping of a UK population: defining the human serum metabolome. Metabolomics : Official journal of the Metabolomic Society 11.

Fei N, Bruneau A, Zhang X, Wang R, Wang J, Rabot S, Gérard P, and Zhao L. 2020. Endotoxin Producers Overgrowing in Human Gut Microbiota as the Causative Agents for Nonalcoholic Fatty Liver Disease. MBio 11. 10.1128/mBio.03263-19

Festi D, Schiumerini R, Eusebi LH, Marasco G, Taddia M, and Colecchia A. 2014. Gut microbiota and metabolic syndrome. World J Gastroenterol 20:16079-16094.

462 Fu J, Bonder MJ, Cenit MC, Tigchelaar EF, Maatman A, Dekens JAM, Brandsma E, Marczynska J, Imhann F, Weersma RK, Franke L, Poon TW, Xavier RJ, Gevers D, 
464

465

466

467

468

469

470

471

472

473

474

475

476

477

478

479

480

481

482

483

484

485

486

487

488

489

Hofker MH, Wijmenga C, and Zhernakova A. 2015. The Gut Microbiome Contributes to a Substantial Proportion of the Variation in Blood Lipids. Circulation research 117:817-824. 10.1161/CIRCRESAHA.115.306807

Fung TC, Olson CA, and Hsiao EY. 2017. Interactions between the microbiota, immune and nervous systems in health and disease. Nature neuroscience 20:145-155. $10.1038 / \mathrm{nn} .4476$

Gevers D, Kugathasan S, Denson LA, Vázquez-Baeza Y, Van Treuren W, Ren B, Schwager E, Knights D, Song SJ, Yassour M, Morgan XC, Kostic AD, Luo C, González A, McDonald D, Haberman Y, Walters T, Baker S, Rosh J, Stephens M, Heyman M, Markowitz J, Baldassano R, Griffiths A, Sylvester F, Mack D, Kim S, Crandall W, Hyams J, Huttenhower C, Knight R, and Xavier RJ. 2014. The treatment-naive microbiome in newonset Crohn's disease. Cell host \& microbe 15:382-392. 10.1016/j.chom.2014.02.005

Goodrich JK, Waters JL, Poole AC, Sutter JL, Koren O, Blekhman R, Beaumont M, Van Treuren W, Knight R, Bell JT, Spector TD, Clark AG, and Ley RE. 2014. Human genetics shape the gut microbiome. Cell 159:789-799. 10.1016/j.cell.2014.09.053

Grundy SM. 2008. Metabolic syndrome pandemic. Arteriosclerosis, thrombosis, and vascular biology 28:629-636. 10.1161/ATVBAHA.107.151092

Hannou SA, Haslam DE, McKeown NM, and Herman MA. 2018. Fructose metabolism and metabolic disease. J Clin Invest 128:545-555. 10.1172/JC196702

He Y, Wu W, Wu S, Zheng H-M, Li P, Sheng H-F, Chen M-X, Chen Z-H, Ji G-Y, Zheng Z-D-X, Mujagond P, Chen X-J, Rong Z-H, Chen P, Lyu L-Y, Wang X, Xu J-B, Wu C-B, Yu N, Xu Y-J, Yin J, Raes J, Ma W-J, and Zhou H-W. 2018a. Linking gut microbiota, metabolic syndrome and economic status based on a population-level analysis. Microbiome 6:172. $10.1186 / s 40168-018-0557-6$

He Y, Wu W, Wu S, Zheng HM, Li P, Sheng HF, Chen MX, Chen ZH, Ji GY, Zheng ZD, Mujagond P, Chen XJ, Rong ZH, Chen P, Lyu LY, Wang X, Xu JB, Wu CB, Yu N, Xu YJ, 
490

491

492

493

494

495

496

497

498

499

500

501

502

503

504

505

506

507

508

509

510

511

512

513

514

Yin J, Raes J, Ma WJ, and Zhou HW. 2018b. Linking gut microbiota, metabolic syndrome and economic status based on a population-level analysis. Microbiome 6:172. $10.1186 / \mathrm{s} 40168-018-0557-6$

He Y, Wu W, Zheng H-M, Li P, McDonald D, Sheng H-F, Chen M-X, Chen Z-H, Ji G-Y, Zheng Z-D-X, Mujagond P, Chen X-J, Rong Z-H, Chen P, Lyu L-Y, Wang X, Wu C-B, Yu N, Xu Y-J, Yin J, Raes J, Knight R, Ma W-J, and Zhou H-W. 2018c. Regional variation limits applications of healthy gut microbiome reference ranges and disease models. Nat Med 24:1532-1535. 10.1038/s41591-018-0164-x

Imhann F, Vich Vila A, Bonder MJ, Fu J, Gevers D, Visschedijk MC, Spekhorst LM, Alberts R, Franke L, van Dullemen HM, Ter Steege RWF, Huttenhower C, Dijkstra G, Xavier RJ, Festen EAM, Wijmenga C, Zhernakova A, and Weersma RK. 2018. Interplay of host genetics and gut microbiota underlying the onset and clinical presentation of inflammatory bowel disease. Gut 67:108-119. 10.1136/gutjnl-2016-312135

Integrative HMP (iHMP) Research Network Consortium. 2019. The Integrative Human Microbiome Project. Nature 569:641-648. 10.1038/s41586-019-1238-8

Johnson CH, Dejea CM, Edler D, Hoang LT, Santidrian AF, Felding BH, Ivanisevic J, Cho K, Wick EC, Hechenbleikner EM, Uritboonthai W, Goetz L, Casero RA, Pardoll DM, White JR, Patti GJ, Sears CL, and Siuzdak G. 2015. Metabolism links bacterial biofilms and colon carcinogenesis. Cell Metab 21:891-897. 10.1016/j.cmet.2015.04.011

Kim B-S, Choi CW, Shin H, Jin S-P, Bae J-S, Han M, Seo EY, Chun J, and Chung JH. 2019. Comparison of the Gut Microbiota of Centenarians in Longevity Villages of South Korea with Those of Other Age Groups. Journal of microbiology and biotechnology 29:429-440. 10.4014/jmb.1811.11023

Kong F, Hua Y, Zeng B, Ning R, Li Y, and Zhao J. 2016. Gut microbiota signatures of longevity. Current biology : CB 26:R832-R833. 10.1016/j.cub.2016.08.015 
515 Konikoff T, and Gophna U. 2016. Oscillospira: a Central, Enigmatic Component of the Human

$516 \quad$ Gut Microbiota. Trends in microbiology 24:523-524. 10.1016/j.tim.2016.02.015

517 Koren O, Spor A, Felin J, Fåk F, Stombaugh J, Tremaroli V, Behre CJ, Knight R, Fagerberg B,

518 Ley RE, and Bäckhed F. 2011. Human oral, gut, and plaque microbiota in patients with

$519 \quad$ atherosclerosis. Proc Natl Acad Sci U S A 108 Suppl 1:4592-4598.

$520 \quad 10.1073 /$ pnas. 1011383107

521 Kraja AT, Vaidya D, Pankow JS, Goodarzi MO, Assimes TL, Kullo IJ, Sovio U, Mathias RA, Sun

522 YV, Franceschini N, Absher D, Li G, Zhang Q, Feitosa MF, Glazer NL, Haritunians T,

523 Hartikainen A-L, Knowles JW, North KE, Iribarren C, Kral B, Yanek L, O'Reilly PF,

524 McCarthy MI, Jaquish C, Couper DJ, Chakravarti A, Psaty BM, Becker LC, Province MA,

525 Boerwinkle E, Quertermous T, Palotie L, Jarvelin M-R, Becker DM, Kardia SLR, Rotter

526 JI, Chen Y-DI, and Borecki IB. 2011. A bivariate genome-wide approach to metabolic

527 syndrome: STAMPEED consortium. Diabetes 60:1329-1339. 10.2337/db10-1011

528 Lau E, Neves JS, Ferreira-Magalhães M, Carvalho D, and Freitas P. 2019. Probiotic Ingestion,

529

530

Obesity, and Metabolic-Related Disorders: Results from NHANES, 1999-2014. Nutrients

531 Liu Z, Bruins ME, Ni L, and Vincken J-P. 2018. Green and Black Tea Phenolics: Bioavailability,

532

533

Transformation by Colonic Microbiota, and Modulation of Colonic Microbiota. J Agric

534 Food Chem 66:8469-8477. 10.1021/acs.jafc.8b02233

Mathur R, Amichai M, Chua KS, Mirocha J, Barlow GM, and Pimentel M. 2013. Methane and hydrogen positivity on breath test is associated with greater body mass index and body

536

537 fat. The Journal of clinical endocrinology and metabolism 98:E698-E702. 10.1210/jc.2012-3144

Morgan XC, Tickle TL, Sokol H, Gevers D, Devaney KL, Ward DV, Reyes JA, Shah SA, LeLeiko 539 N, Snapper SB, Bousvaros A, Korzenik J, Sands BE, Xavier RJ, and Huttenhower C. 
540

541

542 Morotomi M, Nagai F, and Watanabe Y. 2012. Description of Christensenella minuta gen. nov.,

543

544

545

546

547

548

549

550

551

552

553

554

555

556

557

558

559

560

561

562

563

2012. Dysfunction of the intestinal microbiome in inflammatory bowel disease and treatment. Genome biology 13:R79. 10.1186/gb-2012-13-9-r79

sp. nov., isolated from human faeces, which forms a distinct branch in the order Clostridiales, and proposal of Christensenellaceae fam. nov. International journal of systematic and evolutionary microbiology 62:144-149. 10.1099/ijs.0.026989-0

Oki K, Toyama M, Banno T, Chonan O, Benno Y, and Watanabe K. 2016. Comprehensive analysis of the fecal microbiota of healthy Japanese adults reveals a new bacterial lineage associated with a phenotype characterized by a high frequency of bowel movements and a lean body type. BMC Microbiol 16:284.

Perona JS, Schmidt Rio-Valle J, Ramírez-Vélez R, Correa-Rodríguez M, Fernández-Aparicio Á, and González-Jiménez E. 2019. Waist circumference and abdominal volume index are the strongest anthropometric discriminators of metabolic syndrome in Spanish adolescents. European journal of clinical investigation 49:e13060. 10.1111/eci.13060

Peters BA, Shapiro JA, Church TR, Miller G, Trinh-Shevrin C, Yuen E, Friedlander C, Hayes RB, and Ahn J. 2018a. A taxonomic signature of obesity in a large study of American adults. Sci Rep 8:9749. 10.1038/s41598-018-28126-1

Peters U, Suratt BT, Bates JHT, and Dixon AE. 2018b. Beyond BMI: Obesity and Lung Disease. Chest 153:702-709. 10.1016/j.chest.2017.07.010

Plaza-Díaz J, Ruiz-Ojeda FJ, Vilchez-Padial LM, and Gil A. 2017. Evidence of the AntiInflammatory Effects of Probiotics and Synbiotics in Intestinal Chronic Diseases. Nutrients 9. 10.3390/nu9060555

Proctor L. 2019. Priorities for the next 10 years of human microbiome research. Nature 569:623625. $10.1038 / d 41586-019-01654-0$ 
564 Romano KA, Vivas El, Amador-Noguez D, and Rey FE. 2015. Intestinal microbiota composition

565

566

567

568

569

570

571

572

573

574

575

576

577

578

579

580

581

582

583

584

585

586

587

588

589

modulates choline bioavailability from diet and accumulation of the proatherogenic metabolite trimethylamine-N-oxide. MBio 6:e02481. 10.1128/mBio.02481-14

Sanders ME, Merenstein DJ, Reid G, Gibson GR, and Rastall RA. 2019. Probiotics and prebiotics in intestinal health and disease: from biology to the clinic. Nat Rev Gastroenterol Hepatol 16:605-616. 10.1038/s41575-019-0173-3

Segata N, Izard J, Waldron L, Gevers D, Miropolsky L, Garrett WS, and Huttenhower C. 2011. Metagenomic biomarker discovery and explanation. Genome biology 12:R60. 10.1186/gb-2011-12-6-r60

Sonnenburg JL, and Bäckhed F. 2016. Diet-microbiota interactions as moderators of human metabolism. Nature 535:56-64. 10.1038/nature18846

Suliga E, Ciesla E, Głuszek-Osuch M, Rogula T, Głuszek S, and Kozieł D. 2019. The Usefulness of Anthropometric Indices to Identify the Risk of Metabolic Syndrome. Nutrients 11. 10.3390/nu11112598

Ussar S, Griffin NW, Bezy O, Fujisaka S, Vienberg S, Softic S, Deng L, Bry L, Gordon JI, and Kahn CR. 2015. Interactions between Gut Microbiota, Host Genetics and Diet Modulate the Predisposition to Obesity and Metabolic Syndrome. Cell Metab 22:516-530. 10.1016/j.cmet.2015.07.007

Walters W, Hyde ER, Berg-Lyons D, Ackermann G, Humphrey G, Parada A, Gilbert JA, Jansson JK, Caporaso JG, Fuhrman JA, Apprill A, and Knight R. 2016. Improved Bacterial 16S rRNA Gene (V4 and V4-5) and Fungal Internal Transcribed Spacer Marker Gene Primers for Microbial Community Surveys. mSystems 1.

Wang F, Yu T, Huang G, Cai D, Liang X, Su H, Zhu Z, Li D, Yang Y, Shen P, Mao R, Yu L, Zhao M, and Li Q. 2015. Gut Microbiota Community and Its Assembly Associated with Age and Diet in Chinese Centenarians. Journal of microbiology and biotechnology 25:1195-1204. 10.4014/jmb.1410.10014

PeerJ reviewing PDF | (2020:03:46878:3:1:NEW 22 Jun 2020) 
590 Wang L, Jacobs JP, Lagishetty V, Yuan PQ, Wu SV, Million M, Reeve JR, Jr., Pisegna JR, and 591 Tache Y. 2017. High-protein diet improves sensitivity to cholecystokinin and shifts the 592 cecal microbiome without altering brain inflammation in diet-induced obesity in rats. Am $593 \quad$ J Physiol Regul Integr Comp Physiol 313:R473-R486. 10.1152/ajpregu.00105.2017

594 Waters JL, and Ley RE. 2019. The human gut bacteria Christensenellaceae are widespread, 595 heritable, and associated with health. BMC Biol 17:83. 10.1186/s12915-019-0699-4

596 Yang X, and Wang J. 2019. The Role of Metabolic Syndrome in Endometrial Cancer: A Review. $597 \quad$ Frontiers in oncology 9:744. 10.3389/fonc.2019.00744

598 Yu G, Lam TT-Y, Zhu H, and Guan Y. 2018. Two Methods for Mapping and Visualizing

599 Associated Data on Phylogeny Using Ggtree. Molecular biology and evolution 35:3041$600 \quad$ 3043. $10.1093 / \mathrm{molbev} / \mathrm{msy} 194$

601 Ziętak M, Kovatcheva-Datchary P, Markiewicz LH, Ståhlman M, Kozak LP, and Bäckhed F. 602 2016. Altered Microbiota Contributes to Reduced Diet-Induced Obesity upon Cold

603 Exposure. Cell Metab 23:1216-1223. 10.1016/j.cmet.2016.05.001

604 Zmora N, Suez J, and Elinav E. 2019. You are what you eat: diet, health and the gut microbiota. 605 Nat Rev Gastroenterol Hepatol 16:35-56. 10.1038/s41575-018-0061-2

606 


\section{Figure 1}

Bacterial community structure analysis and sequential operational taxonomic units of Christensenellaceae.

(A) PCoA based on unweighted UniFrac distances comparing bacterial community structure of samples between $\mathrm{G} 1$ and G2. Permutational multivariate analysis of variance (PERMANOVA) was carried out to measure effect sizes and significance differences in beta diversity. The threshold of statistical significance was set at $P<0.05$. (B) Visualization of taxa meeting a LDA threshold>3. LEfSe cladogram showed the differential abundant taxa between the two cohorts. Subjects without Christensenellaceae in orange; chris-rich subjects in blue. (C) subOTU at level of Christensenellaceae were arranged according to the read number. 


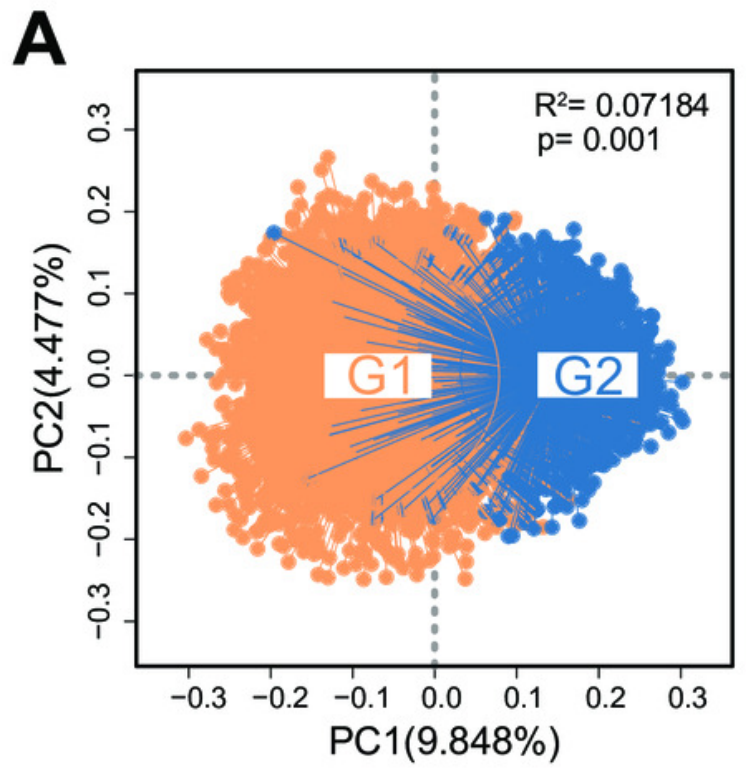

B
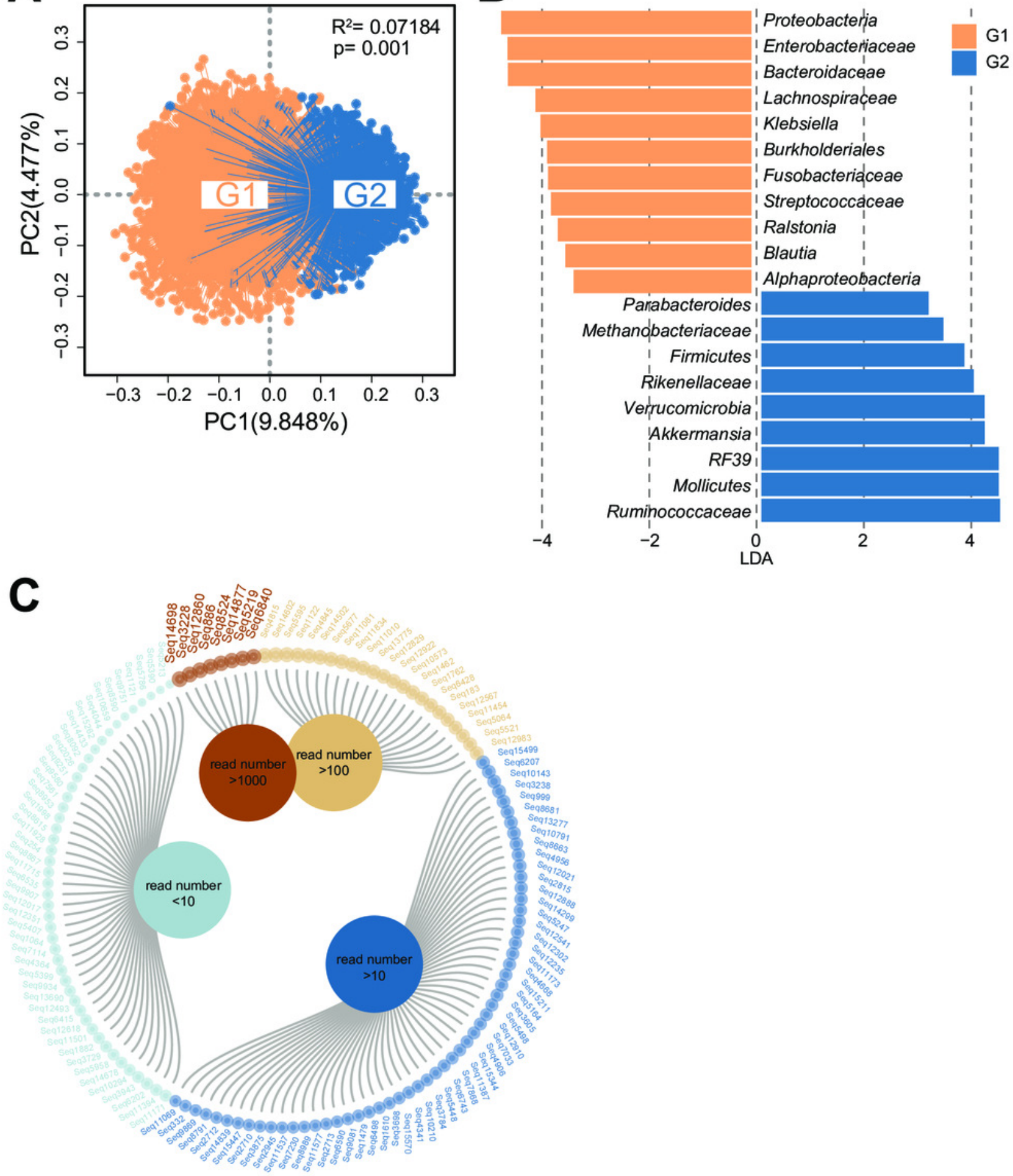


\section{Figure 2}

The geographical features of Christensenellaceae in different regions in GGMP.

(A)Histogram based on the average abundance of Christensenellaceae in 14 regions of

GGMP. City abbreviation is on the X-axis and the abundance value in on the Y-axis. (B)

Annual income and Christensenellaceae abundance in 14 regions of GGMP. (C)The prevalence of metabolic syndrome and the average abundance of Christensenellaceae in each region. The blue line represents metabolic syndrome prevalence and the orange line represents average abundance of Christensenellaceae. (D)Prevalence of metabolic syndrome in people with different Christensenellaceae abundance in each area. Light orange represents $\mathrm{G} 1(\mathrm{n}=3316)$ and light blue represents $\mathrm{G} 2(\mathrm{n}=1465)$. 
A

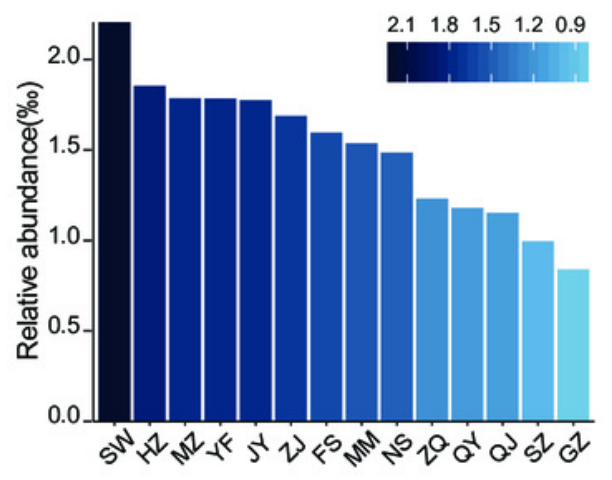

C

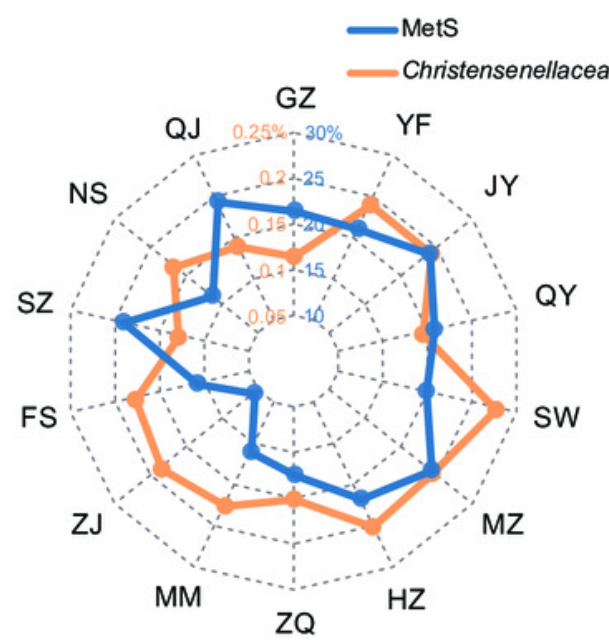

B

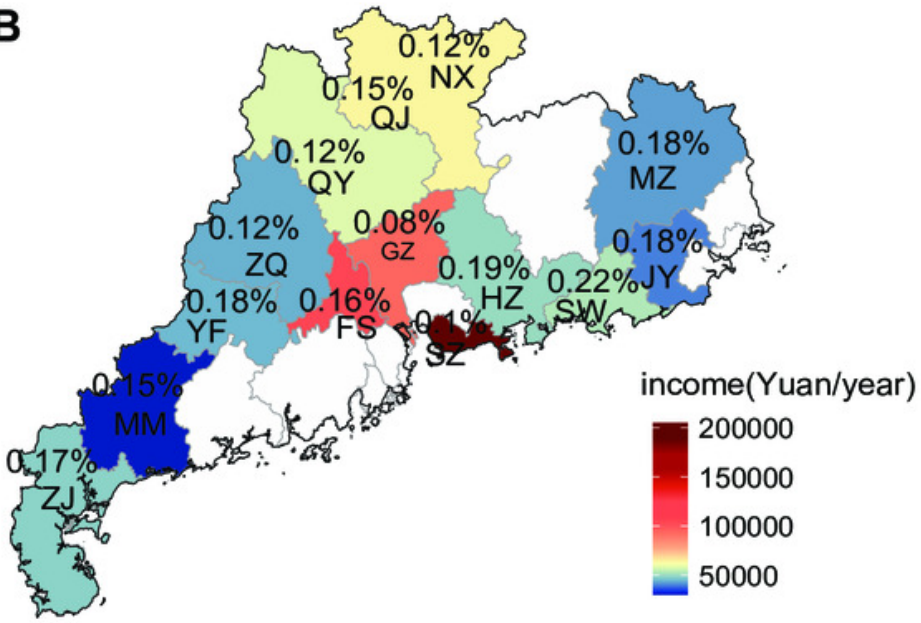

D

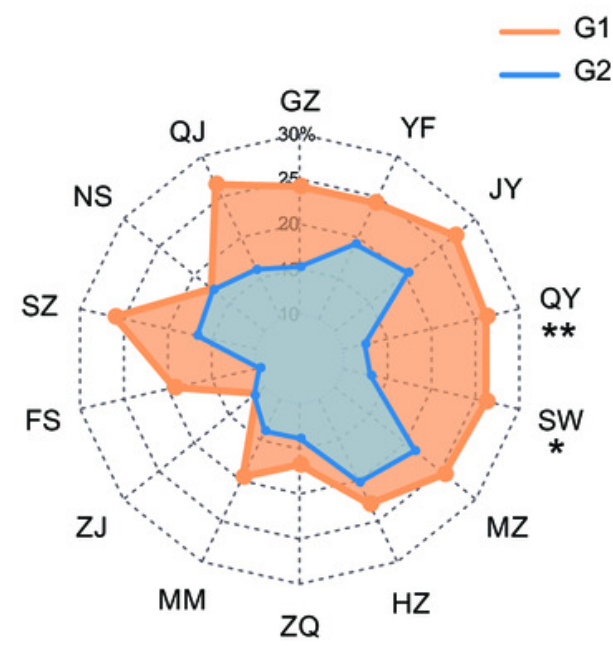




\section{Figure 3}

Metabolic related index and disease status related to Christensenellaceae.

(A)Heatmap of the association between metabolic index and Christensenellaceae as well as its SOTU. Color depth indicates the pearson correlation coefficients. ***P $<0.001$, **P $<0.01$, $* \mathrm{P}<0.05$. (B)The network showed the significant association between Christensenellaceae sub-OTUs and metabolic diseases. The blue arrow represents the negative correlation, and the orange represents positive. The number of significant association was summarized on the edge of network. Mets, metabolic syndrome; FLD, fatty liver disease; HTN, hypertension; USD, urinary system diseases; T2DM, type 2 diabetes.

A

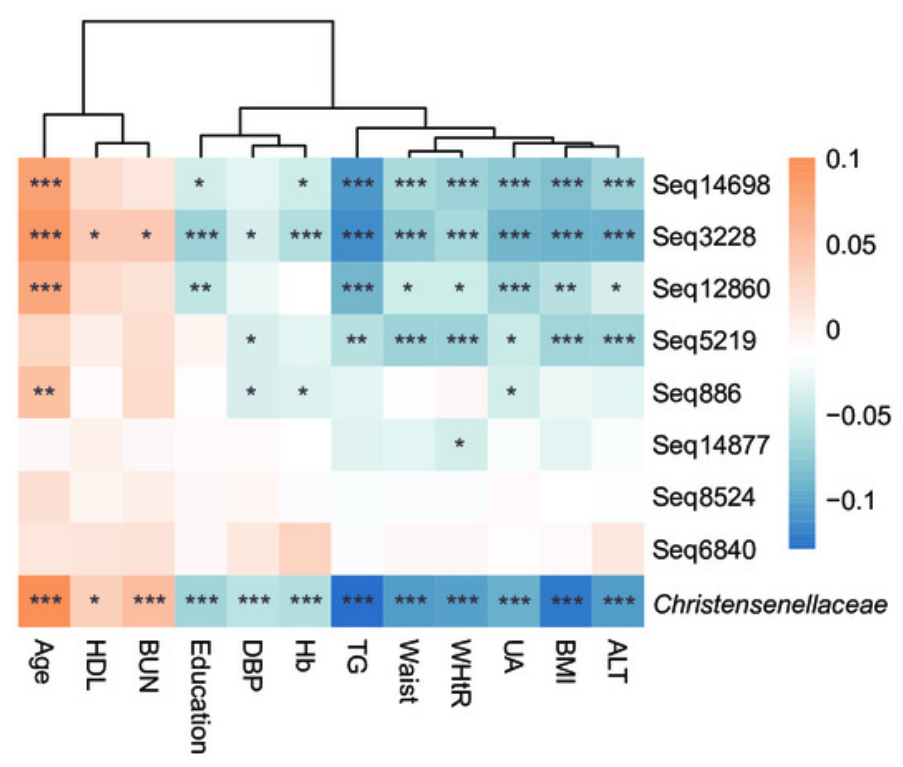

B

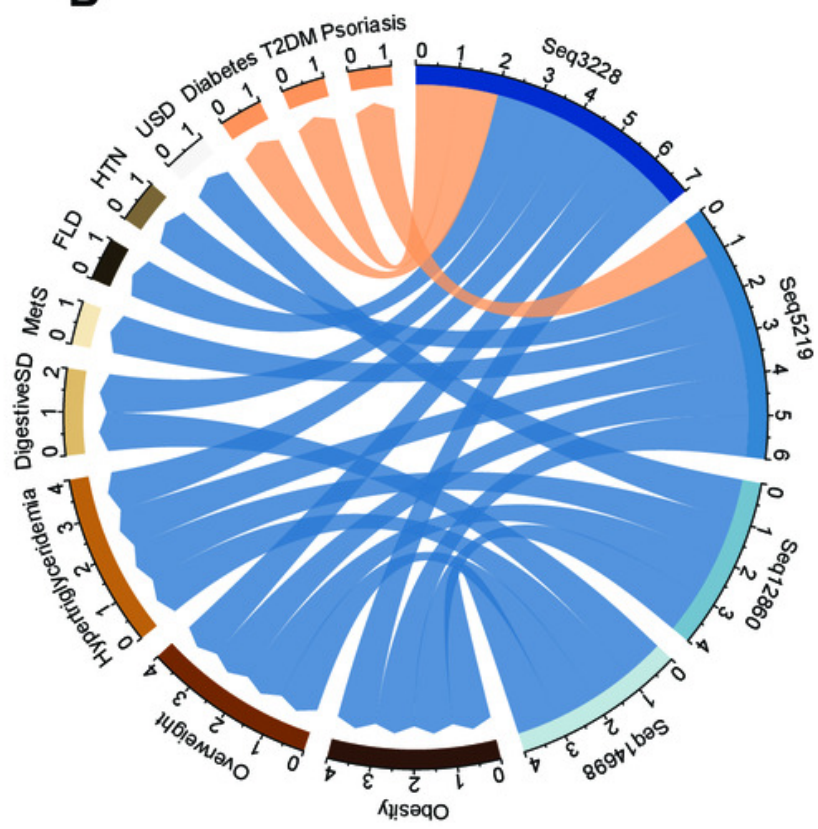




\section{Figure 4}

Microbial taxa associated with Christensenellaceae.

(A) Co-occurrence network between Christensenellaceae and the relative microbiota. The orange line indicates positive correlation and blue negative correlation. $* * \mathrm{P}<0.001, * * \mathrm{P}<$ 0.01 , $* \mathrm{P}<0.05$. (B) Matrix Diagram of sub-OTUs within Christensenellaceae and related microbiota. Microbiota that are significantly associated with Christensenellaceae were plotted. The size of the plots represents the strength of the correlation. 
A
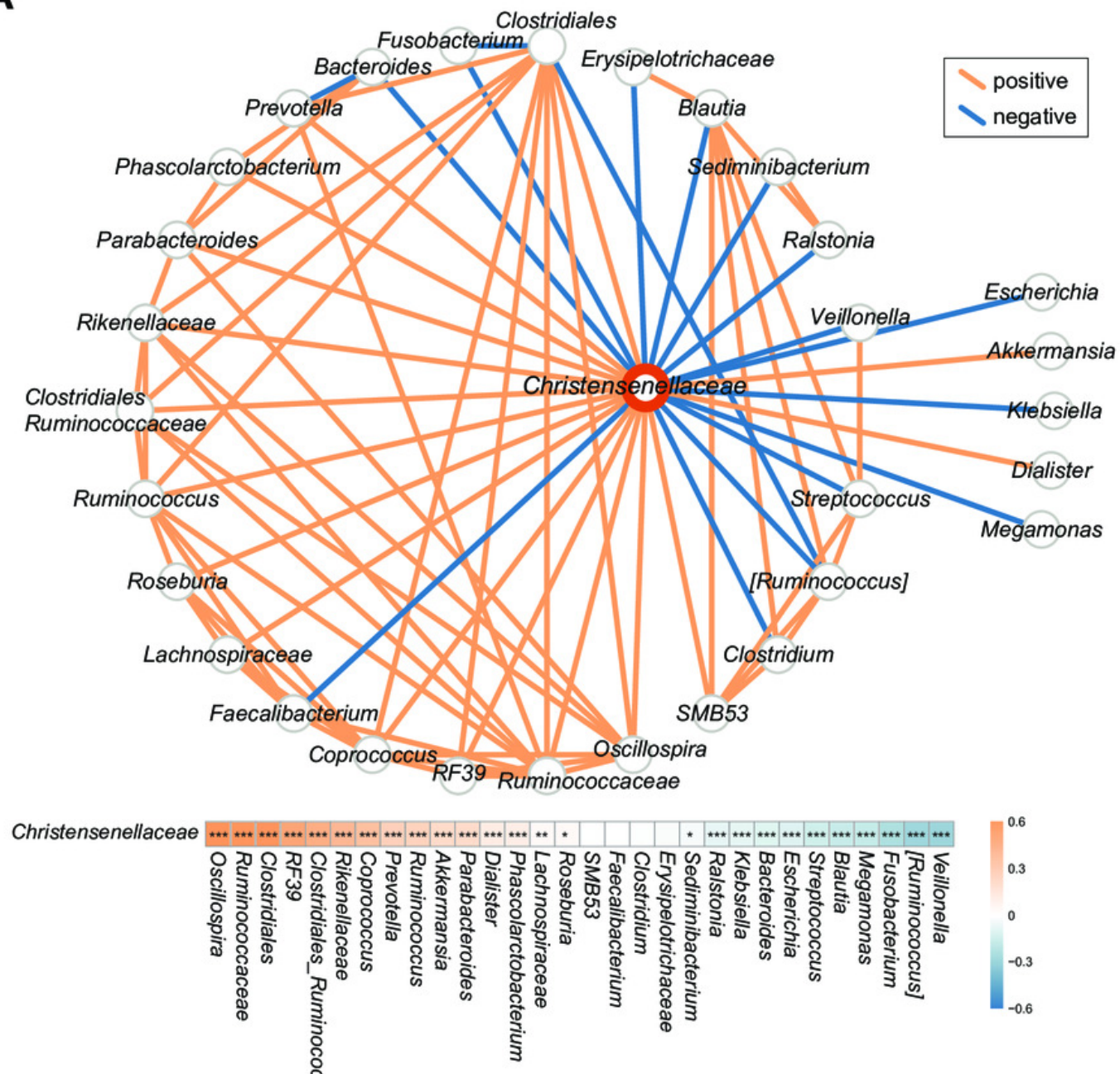

B

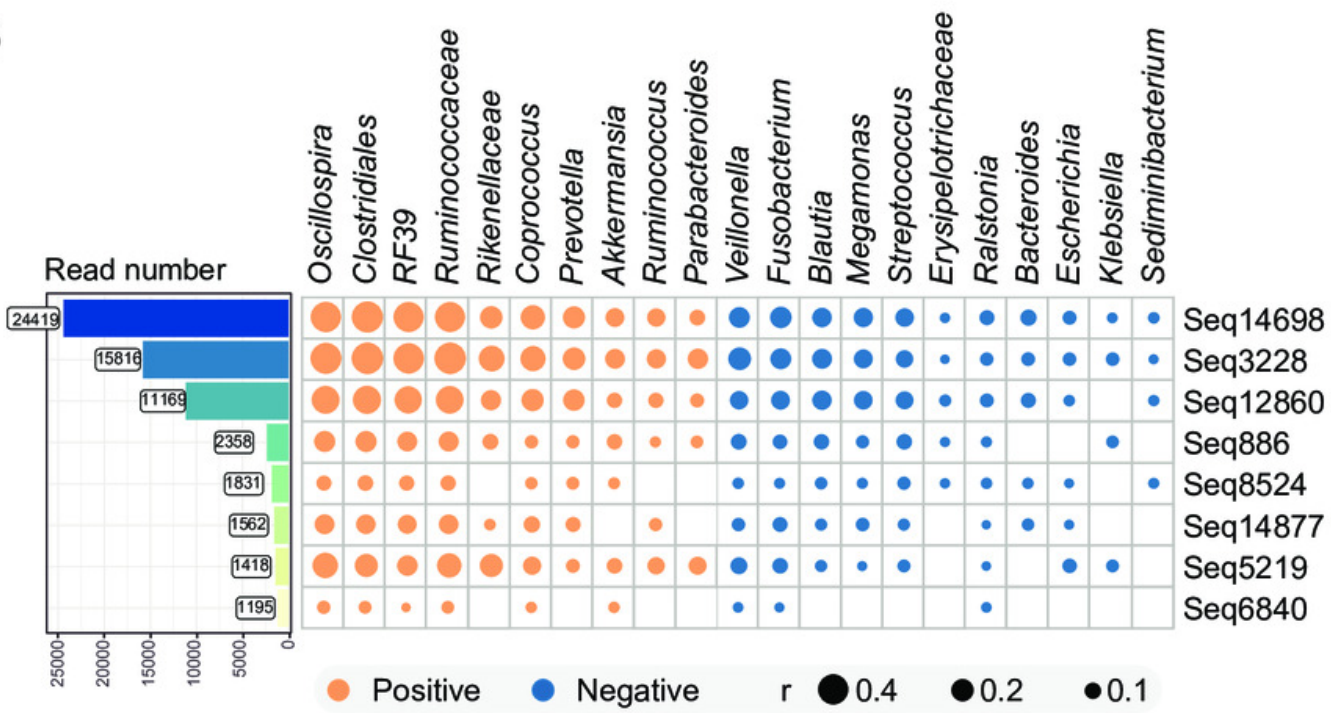




\section{Figure 5}

Differential pathways in individuals with different abundance of Christensenellaceae.

Q1, LDA score<-3, orange color; Q4, LDA>3, blue color.

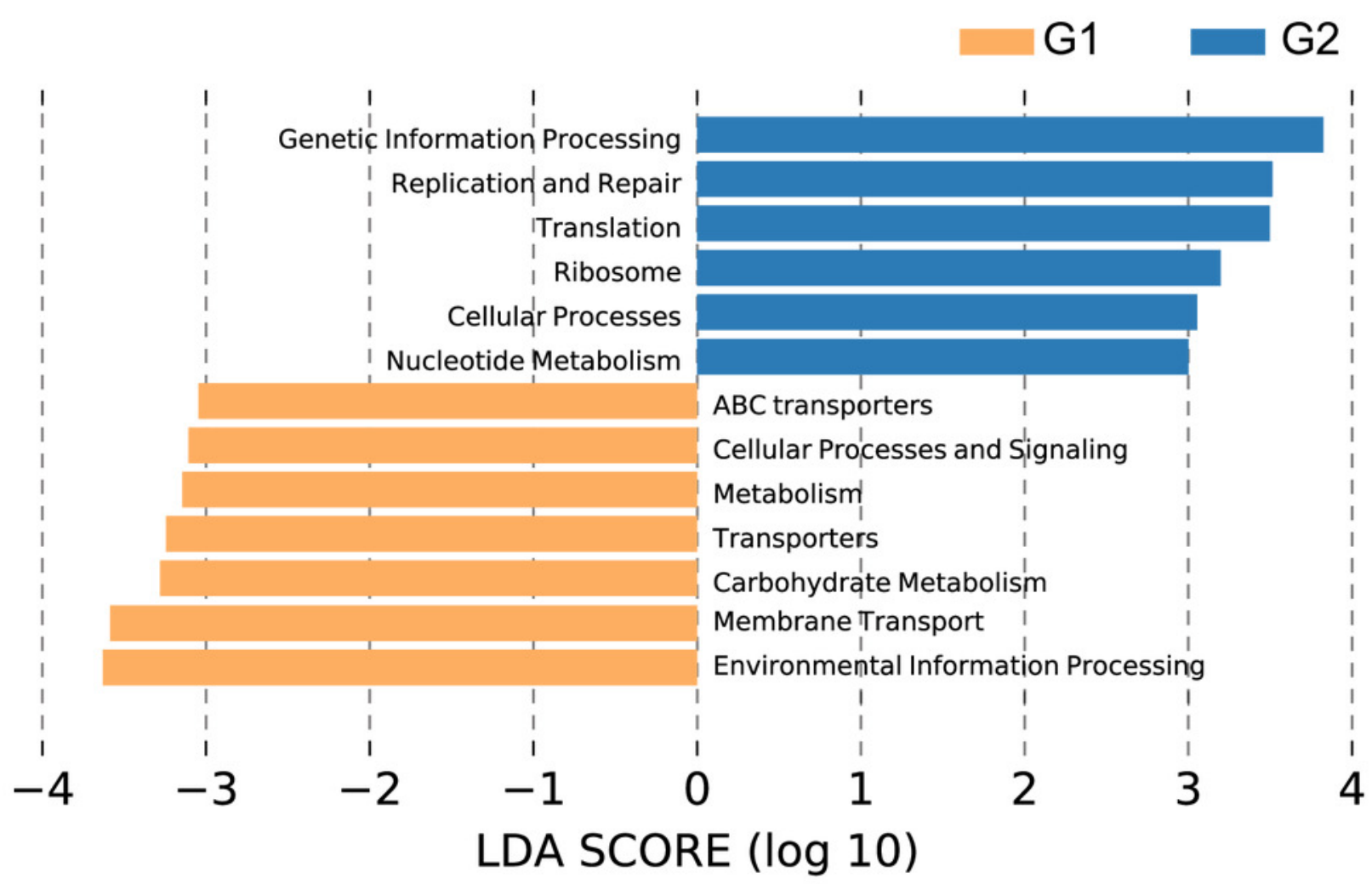




\section{Table $\mathbf{1}$ (on next page)}

Anthropometric parameters and biochemical criterions variations stratified by abundance of Christensenellaceae.

$\mathrm{SD}$, standard deviation; BMI indicates body mass index; WHtR, waist-to-height ratio; UA, uric acid; TG, triglyceride; $A L T$, alanine transaminase; $\mathrm{Hb}$, hemoglobin; $\mathrm{HDL}$, high density lipoprotein. 


\begin{tabular}{|c|c|c|c|}
\hline & $\begin{array}{l}\text { G1-value } \\
(n=3316)\end{array}$ & $\begin{array}{l}\text { G2-value } \\
(n=1465)\end{array}$ & P-value \\
\hline $\begin{array}{l}\text { Age(years) } \\
\text { Median[SD] }\end{array}$ & $53.0[14.5]$ & $55.0[15.2]$ & $<0.001$ \\
\hline $\begin{array}{l}\text { Sex } \\
\text { Female } \\
\text { Male }\end{array}$ & $\begin{array}{l}1830(55.2 \%) \\
1486(44.8 \%)\end{array}$ & $\begin{array}{l}824(56.2 \%) \\
641(43.8 \%)\end{array}$ & 0.517 \\
\hline $\begin{array}{l}\text { BMI }\left(\mathbf{k g} / \mathbf{m}^{2}\right) \\
\text { Median[SD] } \\
\text { Missing }\end{array}$ & $\begin{array}{c}23.4[3.61] \\
39(1.2 \%)\end{array}$ & $\begin{array}{c}22.5[3.23] \\
27(1.8 \%)\end{array}$ & $<0.001$ \\
\hline $\begin{array}{l}\text { Waist(cm) } \\
\text { Median[SD] } \\
\text { Missing }\end{array}$ & $\begin{array}{c}80.4[10.1] \\
39(1.2 \%)\end{array}$ & $\begin{array}{c}78.3[9.22] \\
27(1.8 \%)\end{array}$ & $<0.001$ \\
\hline $\begin{array}{l}\text { WHtR } \\
\text { Median[SD] } \\
\text { Missing }\end{array}$ & $\begin{array}{c}0.512[0.0651] \\
39(1.2 \%)\end{array}$ & $\begin{array}{c}0.497[0.0603] \\
27(1.8 \%)\end{array}$ & $<0.001$ \\
\hline $\begin{array}{l}\text { Obesity } \\
\text { Yes } \\
\text { No } \\
\text { Missing } \\
\end{array}$ & $\begin{array}{c}394(11.9 \%) \\
2883(86.9 \%) \\
39(1.2 \%) \\
\end{array}$ & $\begin{array}{c}92(6.3 \%) \\
1346(91.9 \%) \\
27(1.8 \%) \\
\end{array}$ & $<0.001$ \\
\hline $\begin{array}{l}\text { MetS } \\
\text { Yes } \\
\text { No } \\
\text { Missing }\end{array}$ & $\begin{array}{c}739(22.3 \%) \\
2529(86.4 \%) \\
38(1.1 \%)\end{array}$ & $\begin{array}{c}227(15.5 \%) \\
1209(82.5 \%) \\
29(2.0 \%)\end{array}$ & $<0.001$ \\
\hline $\begin{array}{l}\text { Hypertriglyceridemia } \\
\text { Yes } \\
\text { No } \\
\text { Missing } \\
\end{array}$ & $\begin{array}{c}414(12.5 \%) \\
2864(86.4 \%) \\
38(1.1 \%)\end{array}$ & $\begin{array}{c}99(6.8 \%) \\
1339(91.4 \%) \\
27(1.8 \%)\end{array}$ & $<0.001$ \\
\hline $\begin{array}{l}\text { UA( } \boldsymbol{\mu} \text { mol/L) } \\
\text { Median[SD] } \\
\text { missing }\end{array}$ & $\begin{array}{l}330[94.5] \\
38(1.1 \%)\end{array}$ & $\begin{array}{l}315[87.3] \\
27(1.8 \%)\end{array}$ & $<0.001$ \\
\hline $\begin{array}{l}\text { TG(mmol/L) } \\
\text { Median[SD] } \\
\text { Missing }\end{array}$ & $\begin{array}{l}1.15[1.56] \\
38(1.1 \%)\end{array}$ & $\begin{array}{c}0.9555[1.36] \\
27(1.8 \%) \\
\end{array}$ & $<0.001$ \\
\hline $\begin{array}{l}\text { ALT(U/L) } \\
\text { Median[SD] } \\
\text { Missing }\end{array}$ & $\begin{array}{l}16.0[16.5] \\
107(3.2 \%)\end{array}$ & $\begin{array}{l}14.0[13.7] \\
52(3.5 \%)\end{array}$ & $<0.001$ \\
\hline $\begin{array}{l}\text { Hb(g/L) } \\
\text { Median[SD] } \\
\text { Missing }\end{array}$ & $\begin{array}{l}143[21.9] \\
38(1.1 \%)\end{array}$ & $\begin{array}{l}141[20.3] \\
27(1.8 \%)\end{array}$ & $<0.001$ \\
\hline
\end{tabular}




\begin{tabular}{|l|c|c|c|}
\hline HDL(mmol/L) & & & \\
Median[SD] & $1.22[0.520]$ & $1.25[0.435]$ & 0.0155 \\
Missing & $40(1.2 \%)$ & $27(1.8 \%)$ & \\
\hline
\end{tabular}

1 